Computer-Aided Civil and Infrastructure Engineering,

Volume 36, Issue 9, September 2021, pp. 1185-1202

DOI: $10.1111 /$ mice. 12646

\title{
A Multi-Objective Genetic Algorithm Strategy for Robust Optimal Sensor Placement
}

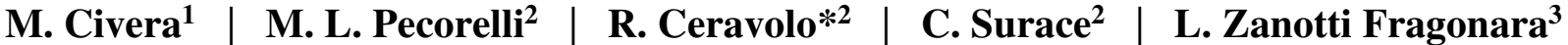

${ }^{1}$ Department of Mechanical and Aerospace Engineering, Politecnico di Torino, Turin, Italy

${ }^{2}$ Department of Structural, Building and Geotechnical Engineering, Politecnico di Torino, Turin, Italy

${ }^{3}$ School of Aerospace, Transportation and Manufacturing, Cranfield

University, Cranfield, United Kingdom

\section{*Correspondence}

Rosario Ceravolo, Politecnico di Torino, Corso Duca degli Abruzzi 24, Turin, 10129, Turin, Italy.

Email: rosario.ceravolo@polito.it

\begin{abstract}
The performance of a monitoring system for civil buildings and infrastructures or mechanical systems depends mainly on the position of the deployed sensors. At the current state, this arrangement is chosen through Optimal Sensor Placement (OSP) techniques that consider only theinitial conditions of the structure. The effects of the potential damage are usually completely neglected during its design. Consequently, this sensor pattern is not guaranteed to remain optimal during the whole lifetime of the structure, especially for complex masonry buildings in high seismic hazard zones. In this paper, a novel approach based on Multi-Objective Optimisation (MO) and Genetic Algorithms (GAs) is proposed for a damage scenario-driven OSP strategy. The aim is to improve the robustness of the sensor configuration for damage detection after a potentially catastrophic event. The performance of this strategy is tested on the case study of the bell tower of the Santa Maria and San Giovenale Cathedral in Fossano (Italy) and compared to other classic OSP strategies and a standard GA approach with a single objective function
\end{abstract}

\section{1 | INTRODUCTION}

Due to the importance that buildings and infrastructures occupy within our society, their structural integrity is not only indispensable to limit possible economic losses, but also fundamental for the safeguard of human lives. Structural Health Monitoring (SHM) is the discipline dedicated to this task. Specifically, vibration-based SHM techniques (such as e.g. in Rafiei \& Adeli (2017)) resort to the dynamic response of the structure of interest to extract useful damage-related features. Real-time online monitoring is particularly essential for frail, valuable buildings of historical and architectural relevance in zones of elevated earthquake risk. One of the most relevant practical issues in this regard concerns the proper placement and direction of the acquisition channels - that is to say, where and in which direction to record the acceleration time histories to better capture the dynamic behaviour of the target structure. This problem goes under the definition of Optimal Sensor Placement (OSP) (Worden \& Burrows, 2001; Gutierrez Soto \& Adeli, 2013; Chang \& Pakzad, 2014). OSP is a well-known, decade-long issue for the dynamic monitoring of any kind of structures ranging from civilian habitations to spacecraft; the task becomes not trivial for real-life structures with complex geometries and different materials involved. Improper sensors placement may generate unreliable data and important changes in the structural responses may go undetected; an optimal layout of the sensor, on the other hand, allows adequate and reliable monitoring (Tondini et al., 2015). Several difficulties arise when OSP principles are translated from the theory to real-life applications. This is particularly true for masonry buildings, where the main difficulties arise from several factors such as the heterogeneous material quality, the large variety of structural components, the limited knowledge of its aged mechanical properties, etc. In these cases, Finite Element (FE) modelling and model updating are the only practical way of dealing with so many practical issues (Chiorino et al., 2011). However, OSP is generally performed only once in a structure's lifetime, achieving a sensor configuration tailormade on its initial conditions. This sensor pattern is not guaranteed to remain optimal when large structural changes occur - e.g., in the case of buildings severely damaged after seismic events (Zanotti Fragonara et al., 2017). A design that does not account for potential damage may fail to capture some relevant changes in the modal components. This would affect noticeably the capability of the monitoring system for early damage detection. Thus, two competing aims exist: the impelling need to deploy a sensor network well-suited for the current structural form and the long-run requirement to make it robust in case of serious damage. The monitoring system is then required to be still operative after damage while being able to 
recognise this novel configuration as a damage condition; these are two distinct tasks which make the problem intrinsically multi-objective.

In this paper, a novel approach based on Multi-Objective Optimisation via a Genetic Algorithm (MOGA) is proposed. Two distinct objective functions (OFs), both concerning the baseline structural configuration and some potential damaged conditions, are set and simultaneously minimised. The obtained sensor pattern represents an optimal compromise between the two instances mentioned above. The approach is tested on the case study of the bell tower of the San Giovenale church in Fossano, Piedmont, Italy, which has been largely damaged by an earthquake in 2012. A predictive FE model, experimentally calibrated accordingly to the current best practices (Bursi \& Wagg, 2009), was utilised to this aim. The damage already present in the current structural condition has been modelled to provide the baseline model; several kinds and extensions of further damage have been considered to simulate the aftermaths of possible future seismic events. The results are then firstly compared to classic OSP strategies (namely, the Eigenvalue Vector Product (EVP), the Effective Independence (EI), and the Average Driving Point Residue (ADPR)) and the sensor pattern currently in use. A second comparison is then carried out against a single cost function-based Genetic Algorithm (SOGA) alternative. The MOGA results are consistent with what the several other well-established techniques returned for the baseline structure and more efficient when damaged conditions are included, even with respect to the simpler single cost function GA. The rest of the article is organised as follows. In Section 2, a preliminary discussion about Optimal Sensor Placement is presented. The OSP problem is described in Section 3; some recalls about the theoretical background of Multi-objective Optimisation are provided as well. Section 4 describes the Case Study of interest. The Results are enlisted and commented in Section 5; the Discussion and Conclusions follow.

\section{2 | METAHEURISTIC ALGORITHMS IN OPTIMAL SENSOR PLACEMENT}

Numerous metaheuristic algorithms have been proposed in the field of OSP and structural dynamics. Trajectory-based algorithms, such as Simulated Annealing (SA), Tabu Search (TS), and other iterated, stochastic or guided local search algorithms have been reported in the literature for structural System Identification (SI) and SHM problems (e.g. in Tong et al. (2014)). Population-based procedures encountered even more success: several variations of Ant Colony (ACO), Particle Swarm Optimisation (PSO), and Differential Evolution (DE) algorithms have all been often applied. Other procedures exist, almost all nature-inspired and derived from some sort of Swarm Intelligence (SI) (e.g. in Sun and Betti (2015)). However, it is difficult to have a completely updated panorama, as new variants are constantly devised; for instance, the parameters self-adaptivity, introduced in Qin and Suganthan (2005) and extensively utilised for SHM applications - see, e.g., Martucci at al. (2018) -, can be arguably considered already overtaken by more recent developments (Zhang and Sanderson, 2009).

In this study, a Genetic Algorithm (GA) was applied. As the name itself clarifies, this family of algorithms mimics the Darwinian selection of the fittest. A classic reference can be found in Goldberg (1989). This kind of metaheuristic has been applied intensively in Civil Engineering and specifically for SHM instances, e.g. by Papadimitriou et al.(2000). GAs were also applied for single- and multi-criteria structural optimisation by Adeli \& Cheng, (1994a); Sarma \& Adeli, (2000a, 2000b). Parallel computing was also found to be especially convenient in this regard by Saleh \& Adeli (1994a, 1994b); Adeli \& Cheng (1994b); Adeli \& Kumar (1995a, 1995b). The interested audience may refer to the book of Adeli \& Sarma (2006). A review of evolutionary algorithms for structural optimisation (including GAs) can be found in Munk et al. (2015). Several improvements were found in more recent years; for instance, Serpik et al. (2017) proposed a genetic algorithm for the optimisation of trusses' shape, size, and topology. Zawidzki \& Jankowski (2019) proposed the multicriterial optimisation of the mechanical and geometrical properties of modular structures. In this work, a MultiObjective (MO) extension of the GA procedure was used, as it will be discussed in more detail in the following Subsection.

\section{1 | Multi-Objective GA Optimisation}

Differently from standard Single-Objective (SO) optimisation, $\mathrm{MO}$ optimisation procedures work by looking for the better compromise between two or more distinct OFs (Deb et al., 2002). For a SO search problem, the three main characteristics are that (i) a best candidate exists, and it is implicit in the model; (ii) the optimisation procedure should discover it or come very close to it; (iii) no supplementary decision making is required after the procedure is completed. In MO, instead, (i) and (ii) remain valid; nevertheless, due to the heterogeneity of objectives, multiple equivalently efficient i.e., Pareto optimal (Censor, 1977) - alternatives may exist. This is more adherent to real-world problems, where the merits of potential solutions involve multiple perspectives and the decision context of the optimisation task is characterised by multiple criteria. However, this is not a constant rule, and a unique solution for the two or more OFs applied may be found. Several MO approaches have been reported in the scientific literature for OSP and Structural Health Monitoring purposes, even if with fundamental differences respect to this work; to the best of the Authors' knowledge, there are no precedents of MOGA approaches applied to several damaged scenarios combined with the structural condition 'as it is'. A very complete and thorough examination of Pareto-optimal sensors 
and excitation layout was carried by Raich and Liszkai (2012). They used an input-output procedure to define the Frequency Response Functions (FRFs) as features of simple case studies, while in this work, an output-only approach and mode shapes have been used. Moreover, they considered two different objectives, i.e., minimising the number of sensors utilised while maximising the diagnostic information collected by the sensor set, imposing several damage scenarios but treating them separately, rather than combining them as done in this work. In this sense, one could decompose a MOGA approach in more single-OF GA tasks, performed in series or added together. However, this will return different results, as will be shown later. Two works closely related to this investigation can be found in Lenticchia et al. $(2017,2018)$. In those cases, SO optimisation was performed, but using a compounded OF. The objective function was made up by both the error in modal analysis for the pristine structure of the Hall $\mathrm{C}$ of Turin Exhibition and the error for the same FE model without infill walls. The aim was the same as this investigation, i.e., to define an OSP criterion robust to damage.

\section{3 | METHODS.}

\section{1 | The OSP Problem.}

It is possible to figure the optimisation task as a decision problem. Consider a discrete grid made up by a finite set of $Q$ potential sensor locations and $R$ acquisition directions for any possible location, both defined a priori. If $R$ is constant for any $Q$, then $N=Q R$ channels are theoretically available. One can either choose to switch on or off any single channel, so there exist only two options for each one. Thus, the problem can be reduced to a combination of yes/no binary decision variables $q_{i j}$, defined as $q_{i j}=1$ if the sensor is allocated at position $j$ and oriented along the $i$-th acquisition direction and 0 otherwise (for $i=1,2, \ldots, R$ and $j=1,2, \ldots, Q$ ). If order does not matter, it is then possible to define the number of all the possible combinations by summing them up. If also the number of the selected sensors is let free to float, this can be done for all the different lengths of the final subset. Hence, only one combination of length zero exists (the empty set). Then, $N$ combinations of one sensor can be considered, $N(N-1) / 2$ subsets of two sensors, and so on, until only one group of $N$ sensors (the full set) remains, for a total of $2^{N}$ possible combinations. The search space can be therefore defined as $\boldsymbol{X} \equiv\{0,1\}^{N} \subset \mathfrak{R}^{N}$.

Being the set of candidate solutions finite, it can theoretically be solved by brute force, testing all the possible combinations. Yet this is not practically doable in real-life cases, where the FE models of large-scale structures can have hundreds or thousands of degrees of freedom, due to limited computational capability (Adeli \& Saleh, 1997). Even if viable, a complete sampling of the search space would never be the most efficient approach. Indeed, the optimisation task is not verifiable in polynomial time, as its complexity grows exponentially - being $O\left(2^{N}\right)$. In the example of the case study analysed here, 31 different options make up the complete set of the possible (arbitrarily) pre-selected locations. For any location, there are two possible directions for recording, along the two horizontal $\mathrm{x}$ - and $\mathrm{y}$ - axes (the vertical response along with the $\mathrm{z}$-axis being of limited interest). This makes up $N=62$ channels and circa $4.61 \cdot 10^{18}$ possible combinations.

In principle, any sort of search algorithm (i.e., any heuristic procedure), can be used to explore the search space partially and to find a near-optimal solution. However, metaheuristic approaches are, by general consensus, the aptest strategy from a cost/benefit point of view. Even if their results cannot be proved mathematically to be optimal, it is a fact recognised by decades of intensive use that their sub-optimality is generally limited, and the returned combination is never too distant, in the search space, from the actual optimum. For these reasons, they have been proposed for the OSP of historical masonry structures with localised cracks and/or diffuse material deterioration (Barontini et al., 2017).

\subsection{Theoretical Definition of the MO Procedure.}

The MO procedures are still an area of current research, but it is known that non-dominated solutions can be approximated with good results through population-based meta-heuristics. Some basic concepts about the MO theoretical background are here briefly recalled to provide the context of this work; one can find a more detailed discussion in any related textbook such as e.g. Antunes and Alves (2016). Everything is presented here for a maximisation problem (considering fitness functions) but can be easily mirrored to minimisation problems (assuming cost functions, as actually done here and often occurring in practice). The MO optimisation process is equivalent to mapping the $\mathrm{N}$ dimensional decision variable space $\boldsymbol{X} \subseteq \mathfrak{R}^{N}$, where all potential solutions are confined, onto the objective function space $\boldsymbol{Z} \equiv\left\{\boldsymbol{f}(\boldsymbol{x}) \in \mathfrak{R}^{p}: \boldsymbol{x} \in \boldsymbol{X}\right\}$, defined by $p \geq 2$ OFs. The objective functions dimensionality is generally much lower than the dimensionality of the decision variables (i.e. there are much more variables than OFs, $N \gg p$ ). Thus, each potential candidate $\boldsymbol{x} \in \boldsymbol{X}$ has an image vector $\boldsymbol{z}:=\boldsymbol{f}(\boldsymbol{x})$, whose components $z_{i}=f_{i}(\boldsymbol{x}), i=1,2, \ldots p$, are the values of each corresponding $\mathrm{OF}$ for that point of the feasible region. The MO optimisation can, therefore, be also referred to as vectorial optimisation, as the more than one OFs are arranged into a vector, which is the quantity to be optimised. This can lead to one or more non-dominated solutions, each one corresponding to a vector composed of the respective $\mathrm{OF}$ values. Hence, the optimisation task can be formulated as

$$
\max _{\boldsymbol{x}}\left(f_{1}(\boldsymbol{x}), f_{2}(\boldsymbol{x}), \ldots, f_{p}(\boldsymbol{x})\right) .
$$

If $\boldsymbol{X}$ is non-empty and a maximum for all the OFs being maximised exists and is included in $\boldsymbol{X}$, the frontiers of 
feasibility for $\boldsymbol{Z}$ can be defined by mapping the edge of such an enclosed region. The non-dominated components of the sodefined frontier of $\boldsymbol{Z}$ form what is generally known as the Pareto front. Non-domination is here assumed as a synonym of non-inferiority, i.e., it requires that no other feasible solution improves simultaneously all OFs. Any non-dominated / noninferior solution in the objective function space corresponds to an equivalently efficient solution in the decision variable space. This ensemble can be analytically described as:

$$
Z_{E}:\left\{z=f(x) \in Z: x \in X_{E}\right\},
$$

which is the analytical definition of the Pareto front. $\boldsymbol{X}_{\boldsymbol{E}}$ indicates the set of the efficient solutions; a general solution $\boldsymbol{x}^{\prime}$ is considered efficient if there are no other feasible solution $\boldsymbol{x}$ such that $f_{k}(\boldsymbol{x}) \geq f_{k}\left(\boldsymbol{x}^{\prime}\right)$ for all $k=1,2, \ldots p$ and being the inequality strict for at least one $k$, i.e. $f_{k}(\boldsymbol{x})>f_{k}\left(\boldsymbol{x}^{\prime}\right)$. All the other options are, by definition, (Pareto) dominated and would result in a non-efficient, sub-optimal sensor placement. In particular, if the domain is discrete (as it is in this case), it is possible to define a nondomination ranking, such as that rank 1 solutions are non-dominated or dominated by another rank 1 individuals only; rank 2 elements are dominated only by themselves or by rank 1 solutions; and so on (a general rank $k$ element is therefore dominated by all rank $k-1$ or lower elements, at least, and by all the other rank $k$ combinations or lower, at most). It is also possible to distinguish between weakly and strictly non-dominated solutions; in the first case, only a subset of all dimensions is non-dominated. In a 2dimension problem, this can only happen if one dimension is dominated, while the other is inferior to other points belonging to the frontier of the feasibility region in $\boldsymbol{Z}$; their equivalent points in $\boldsymbol{X}$ are denominated weakly efficient solutions.

\section{3 | The Two Cost Functions}

The most critical choice for any optimisation procedure is the definition of one or more appropriate objective functions. The sum of the off-diagonal elements of the Modal Assurance Criterion (MAC) matrix is one of the most recurrent approaches for the quantitative measure of the goodness-of-fit of a sensor network and is often used in combination with GA, as e.g. in Yi et al. (2011)). In formal terms, the MAC between any two mode shapes $\boldsymbol{\varphi}_{A}, \boldsymbol{\varphi}_{B}$ can be stated as

$$
\operatorname{MAC}\left(\boldsymbol{\varphi}_{A}, \boldsymbol{\varphi}_{B}\right)=\frac{\left|\boldsymbol{\varphi}_{A}^{T} \boldsymbol{\varphi}_{B}\right|^{2}}{\left(\varphi_{A}^{T} \varphi_{A}\right)\left(\boldsymbol{\varphi}_{B}^{T} \boldsymbol{\varphi}_{B}\right)}
$$

and can assume any value in between zero (no correlation) and 1 (perfect correlation). From Eq (3) several variants have been developed over the years (Allemang, 2003). The two of interest here are the AutoMAC and the Cross-MAC. The difference lies only in that the AutoMAC compares the modes of a structure among themselves, while Cross-MAC compares the modes of two different structures or the same structure in two different structural configurations. These two definitions are here used to reflect the two criteria. Consider a baseline structure and $M$ known, possible damaged configurations of the same, supposedly caused by a seismic event. It is possible to consider $N_{m}$ mode shapes for each configuration. For the baseline model, that means $\boldsymbol{\Phi}^{\text {base }}=\left[\begin{array}{llll}\varphi_{1}^{b} & \varphi_{2}^{b} & \ldots & \varphi_{N_{m}}^{b}\end{array}\right]$. The same applies to the first damage scenario $\boldsymbol{\Phi}^{D 01}$, and so on. The set of all the mode shapes of all configuration is then given by

$$
\Phi=\left[\begin{array}{llll}
\Phi^{\text {base }} \Phi^{D 01} \Phi^{D 02} \ldots \Phi^{D M}
\end{array}\right],
$$

such that $\boldsymbol{\Phi}_{p}^{\mathbf{1}}=\boldsymbol{\varphi}_{p}^{b}$ is the $p$-th mode shape of the baseline structure and $\boldsymbol{\Phi}_{\boldsymbol{q}}^{\mathbf{j}+\mathbf{1}}=\boldsymbol{\varphi}_{\boldsymbol{q}}^{\boldsymbol{D} \mathbf{j}}$ is the $q$-th mode shape of the $j$-th damage scenario $(j=1,2, \ldots, M)$. Consider that the mode shapes contained in $\boldsymbol{\Phi}$ are defined from the whole set of the 62 channels from the calibrated FE model; $\widehat{\boldsymbol{\Phi}}$ is then made up by the same components as $\boldsymbol{\Phi}$ but this time, only considering an arbitrarily large subset of the channels available, so that the components that correspond to the omitted channels are zeroed. The main aims are, therefore, (I) to have $\widehat{\boldsymbol{\Phi}} \cong \boldsymbol{\Phi}$ for the desired number of channels - i.e., the classic OSP problem, but extended to include the damaged configuration -, and (II) to be able to discern the set of mode shapes in $\widehat{\boldsymbol{\Phi}}^{\text {base }}$ from any other set $\widehat{\boldsymbol{\Phi}}^{\boldsymbol{D} \mathbf{j}}$ - i.e., the classic mode shape-based Structural Health Monitoring problem. It is thus possible to mathematically formulate the generic term in position $(p, q)$. For the $M+1$ AutoMAC matrices, this becomes

$$
\operatorname{MAC}\left(\boldsymbol{\Phi}_{p}^{i}, \widehat{\boldsymbol{\Phi}}_{q}^{i}\right)=\frac{\left|\boldsymbol{\Phi}_{p}^{i^{T}} \widehat{\boldsymbol{\Phi}}_{q}^{i}\right|^{2}}{\left(\boldsymbol{\Phi}_{p}^{i^{T}}{ }^{2} \boldsymbol{\Phi}_{p}^{i}\right)\left({\boldsymbol{\Phi}_{q}^{i}}^{{ }^{T}} \widehat{\Phi}_{q}^{i}\right)}
$$

for $i=1,2, \ldots, M+1$, while for the $M$ Cross-MAC matrices between the baseline model and any $j$-th damage scenario it is

$$
\operatorname{MAC}\left(\widehat{\boldsymbol{\Phi}}_{p}^{\mathbf{1}}, \widehat{\boldsymbol{\Phi}}_{q}^{\mathbf{j}+\mathbf{1}}\right)=\frac{\left|\widehat{\boldsymbol{\Phi}}_{p}^{\mathbf{1}^{T}} \widehat{\boldsymbol{\Phi}}_{q}^{\mathbf{j}+\mathbf{1}}\right|^{2}}{\left(\widehat{\boldsymbol{\Phi}}_{p}^{\mathbf{1}^{T}} \widehat{\boldsymbol{\Phi}}_{p}^{\mathbf{1}}\right)\left(\widehat{\boldsymbol{\Phi}}_{q}^{\mathbf{j}+\mathbf{1}^{T}}{\widehat{\boldsymbol{\Phi}_{q}^{j+1}}}^{\mathbf{j}+\mathbf{1}}\right)}
$$

for $j=1,2, \ldots, M$. The multi-optimisation $\mathrm{GA}$ is here performed simultaneously as the minimisation of the offdiagonal terms of the (normalised and weighted) AutoMACs, named here $f_{1}$, and of all terms, diagonal and off-diagonal as well, of the Cross-MACs, again normalised and weighted. This second function is named $f_{2}$. The rationale is quite straightforward. Consider the baseline and the $M$ damaged AutoMACs. These are all supposedly diagonal for an optimal (orthogonal) set (Worden \& Burrows, 2000). Hence, minimising the off-diagonal terms assures an optimal sensor placement on the current structure and the damaged scenarios. As mentioned above, the AutoMAC is a classic OSP strategy, the main novelty resides in (1) its parallel optimisation on several structural conditions at once and (2) its pairing with $f_{2}$ in a MO optimisation framework. On the other hand, for the $M$ Cross-MACs, the minimisation of the off-diagonal terms 
guarantees, again, a clear distinction between different modes; while by minimising also the diagonal terms, the modal distinction with respect to the current structural configuration is ensured, such that the so-optimised sensor placement distinguishes at its best the baseline and damaged mode shapes. This enforces that $\boldsymbol{\Phi}^{b} \neq \boldsymbol{\Phi}^{D} \mathbf{j} \Rightarrow \widehat{\boldsymbol{\Phi}}^{\boldsymbol{b}} \neq \widehat{\boldsymbol{\Phi}}^{\boldsymbol{D} \mathbf{j}}$. Note that, differently from the AutoMACs, this second task is performed exclusively considering the selected channels, denoted by the hat symbol ' $\wedge$ ', since there is no need to compare the whole baseline and damaged mode shapes, which are known to differ from each other. The two functions can be written analytically as

$$
f_{1}(\boldsymbol{x})=\sum_{i=1}^{M+1} \frac{w(i)}{\mathrm{n}_{1}} \sum_{p=1}^{N_{m}} \sum_{\substack{q=1, p \neq q}}^{N_{m}} M A C\left(\boldsymbol{\Phi}_{\boldsymbol{p}}^{\boldsymbol{i}}, \widehat{\boldsymbol{\Phi}}_{\boldsymbol{q}}^{\boldsymbol{i}}\right)
$$

and

$$
f_{2}(\boldsymbol{x})=\sum_{\mathrm{j}=1}^{M} \frac{w(j)}{\mathrm{n}_{2}} \sum_{p=1}^{N_{m}} \sum_{q=1}^{N_{m}} M A C\left(\widehat{\boldsymbol{\Phi}}_{\boldsymbol{p}}^{\mathbf{1}}, \widehat{\boldsymbol{\Phi}}_{\boldsymbol{q}}^{\mathbf{j}+\mathbf{1}}\right)
$$

where $N_{m}$ and $M$ are set to 10 and 12 in this study. $\mathrm{n}_{1}=N_{m}$. $\left(N_{m}-1\right)$ and $\mathrm{n}_{2}=N_{m}^{2}$ are utilised to normalise the error functions between 1 (worse condition) and 0 (ideal optimum). The weights in the array $w$ are all varied between $[0,1]$ to express the significance attributed to each specific scenario (baseline included). Since the envisioned damage scenarios reflect some real crack patterns as surveyed in similar high-rise masonry building, it is also possible to calibrate the weighting factors accordingly to their expected probability. This point will be better detailed in the next Sections.

It must be said that it would be possible to define $M$ OFs as $f_{2}$ and further $M+1$ OFs as $f_{1}$ for $M$ damage scenarios. However, adding too many OFs would make the problem a socalled Many Objective Optimisation (Ishibuchi et al., 2008), which is inherently more challenging to handle, due to difficulties in the scalability of the algorithms, and without the certainty of effective improvements in the results. Finally, the MOGA problem becomes

$$
\min _{\boldsymbol{x}}\left(f_{1}(\boldsymbol{x}), f_{2}(\boldsymbol{x})\right),
$$

while for the SOGA, it was used the sum of the two functions, similar to what has been done in Lenticchia et al.(2017). and Lenticchia et al. (2018), i.e.

$$
\min _{\boldsymbol{x}}\left(f_{1}(\boldsymbol{x})+f_{2}(\boldsymbol{x})\right)
$$

In Eq (9), the use of a MOGA approach allows keeping distinct the Cross-MACs from the AutoMACs. Noteworthy, the two OFs are closely related and share a common measurement unit, thus no incommensurability issue arises. Results were compared against several well-established, nonstochastic methods as well. Both energy-based and information matrix-based approaches were considered, as they are experimentally known to produce comparable and satisfactory results in real-life large structures. The Eigenvalue Vector Product (EVP) (Doebling, 1995), the Average Driving Point Residue (ADPR) (Penny et al., 1994), and the Effective Independence (EI) (Kammer, 1991) were all considered. The ADPR method selects the grid points with the highest average driving point residue, defined as (for $N_{m}$ vibrational modes)

$$
\operatorname{ADPR}_{\mathrm{j}}=\sum_{i=1}^{N_{m}} \frac{\varphi_{i j}^{2}}{\omega_{i}}
$$

referred to the $j$-th coordinate of the $i$-th eigenvector $\varphi$ (and respective natural frequency $\omega$ ). The rationale is that the selected coordinates are the ones which contribute the most to the mode shape. The EVP value is defined at any coordinate by the product of the eigenvector component at that grid point, for all the modes considered (Worden \& Burrows, 2001), that is to say,

$$
\mathrm{EVP}_{\mathrm{j}}=\prod_{i=1}^{N_{m}} \varphi_{i j}
$$

the coordinates with the highest EVP are then considered. Finally, the EI algorithm is iterative and works by deleting, at any iteration, the acquisition channel with the lowest fractional contribution to the independence of the target modes. In turn, these fractional contributions correspond to the terms on the diagonal of the matrix

$$
\mathrm{E}=\Phi \cdot\left\{\Phi^{\mathrm{T}} \Phi\right\}^{-1} \cdot \Phi^{\mathrm{T}},
$$

where $\Phi$ is the matrix of the target modes. In this case, the concept is that the procedure tends to maintain unvaried the determinant of the Fisher Information Matrix (FIM), thus preserving information while deleting the less relevant channels. This approach is not too dissimilar from one of the two methods investigated. by Reichert er al (2019). The EI approach is arguably one of the most used methods for benchmarking novel proposal in OSP as its theory has been deeply investigated throughout the years (Li et al., 2007).

\subsection{The MO Genetic Algorithm}

The elitist MO Genetic Algorithm specifically used here is an implementation of the well-known Non-dominated Sorting Genetic Algorithm II (NSGA-II) (Deb et al., 2002) which is an improved version of the NSGA method (Srinivas and Deb, 1994) applied by Raich \& Liszkai (2012). With respect to the NSG Algorithm, NSGA-II includes an elitist strategy to speed up the process and is computationally less expensive, plus other ameliorations. NSGA-II was also recently proved by Ciro et al. (2016) to be a benchmark for Multi-Objective Optimisation with few OFs (e.g. 2, as here) and successfully applied for structural optimisation by Zawidzki and Jankowski (2019). The specific details of the algorithm can be found in the original 
work of Deb et al. (2002); here, the main steps are briefly reported for completeness. The algorithm consists of three phases: Initialisation, Iterations, and Ending. Specifically:

1. The Initialisation phase defines the initial population of candidates.

2. The Iteration phase can be split into eight sub-steps, that is to say: (a) parents selection via binary tournament selection; (b) children generation via gene mutation and crossover; (c) scoring of the children combinations according to their OFs values; (d) definition of the extended population matrix; (e) computation of the domination ranks among all the elements in the extended population matrix; (f) computation of the crowding distance $d_{r}$ (see Deb et al. (2002) in the OF space by ranks, for all elements in all ranks; $(\mathrm{g})$ trimming of the extended population matrix to match the number of elements of the pre-set population; (h) the maximal finite crowding distance measure among the points of rank $1 d$ is calculated. The loop is interrupted when one of the stopping criteria is reached.

3. The Ending phase returns the selected winner(s) of the genetic competition, or the remaining population of the last generation if the maximum number of generations is exceeded, if no feasible combination is found, or if, considering some last consecutive generations, the change of spread in the Pareto Front stalls (Rudenko \& Schoenauer, 2004).

The specific settings used in this study are as follow. For the Initialisation phase, the population size was set to 50 with all elements selected via uniform random sampling. The procedure was run 52 times with the number of channels to be included ranging from 10 to 62 . Regarding (step b) of the Iteration phase, a uniform crossover was applied between two parents, as this technique is proved to show the best exploration characteristics among the common crossover options, also for structural optimisation purposes (Hasançebi \& Erbatur, 2000). The crossover rate was set at $80 \%$. Gaussian gene mutation was enforced with a mutation rate of 0.01 , which was considered small enough to exploit the regions found by the crossover operator. Regarding (step g), an elite group of individuals, set equal to $35 \%$ of the elements included in the Pareto front at that iteration, was guaranteed to survive to the next generation). For the ending phase, the number of maximum generations was set to 400 . The performances of the code were tested on two well-known benchmark problems, the test function \#1 from Schaffer (1985) and the test case \#2 from Binh \& Korn (1997). In both cases, results comparables to the ones reported by the original papers were found after 192 and 286 generations, respectively.

\section{4 | THE CASE STUDY}

The bell tower of the Santa Maria and San Giovenale Cathedral in Fossano (Italy) was built in the XIV century. The bell tower reaches an overall height of 46 meters considering the octagonal belfry on its top and has a square cross-section; the thickness of the wall is constantly 1.5 meters up to $35 \mathrm{~m}$ and decreased at 0.5 meters at the belfry. In the whole structure, three slabs are present. The first one (at $9.9 \mathrm{~m}$ from the ground floor) is made of masonry. The second one is at $28.2 \mathrm{~m}$ and made of wood. The last one separates the main structure from the belfry at level $35 \mathrm{~m}$ and it is a composite slab, with a lower web of arched beams and an upper layer made of wood. The bell tower (depicted in Figure 1.a, on the left) suffered large seismic damage along the centuries and it is currently reinforced with 11 levels of steel ties-rod systems on the external façades. Its current structural condition - which is already damaged - is referred to as the 'baseline' condition in this study.

The FE model of the structure 'as it is' has been developed on ANSYS ® Mechanical APDL TM, compounded by 7439 elements (quadrangular 8-Node SHELL 281 where preferably applied wherever possible) and totalling 15233 nodes. To better reconcile the dynamic response of the model to the experimental evidence, considering that the mechanical properties of the building materials are non-homogeneous and that they are locally deteriorated, the model has been subdivided into six macro-elements. As represented in Figure 1.a (on the right side), the mechanical properties of each macro area (Young's modulus $E$, Poisson ratio $v$, and density $\delta$ ) have been calibrated separately to match the behaviour of the structure, based on the acquired vibrational data and on a core drilling campaign as described in Ceravolo et al. (2016). It can be noticed that levels \#1 and \#2 were found to be less resistant due to poorer building material and thus more prone to develop damage in the future. The connection walls with the attached church of San Giovenale were modelled with a fictitious material with $E=500 \mathrm{MPa}$ to simulate the weak connection state with the nearby church, as evidenced by the in-situ survey (Ceravolo et al., 2016). The damage was modelled by reducing the equivalent Young's modulus of specific elements; this is a common procedure, commonly used for considering the reduced properties of cracked masonry of structures in postearthquake conditions. More details about the modelling of the damage, also according to experimental investigations, can be found in Ceravolo et al. (2016). An example of the recorded acceleration time history is depicted in Figure 1.b as captured by the current sensor network using ambient vibrations (road traffic, wind, microtremors etc) as an input excitation. Uniaxial PCB Piezoeletronics capacitive accelerometers (Model 3701G3FA3G; see Figure 1c,) are utilised. Each one is characterised by a resolution of $30 \mu \mathrm{g}$, a sensitivity of circa $1 \mathrm{~V} / \mathrm{g}$, and a measuring range between 0 and $3 \mathrm{~g}$. The monitoring system includes also a Difa-LMS SCADAS data logger and a 
laptop hard-drive with the acquisition software. The modal parameters were then extracted from the accelerometric data using a stochastic subspace identification algorithm developed at the Department of Structural, Geotechnical and Building Engineering of Politecnico di Torino (Ceravolo \& Abbiati 2013).

For the sake of this study and some previous works (Ferraris et al., 2020; Civera et al., 2019), 12 hypothetical damage scenarios were considered. The pattern of the damaged configurations, enlisted in Table 1, comes from the personal experience of the Authors and from the most recurrent crack patterns and failure modes of masonry towers (especially the medieval ones) found in the literature; it is therefore considered descriptive of typical post-earthquake effects on similar masonry tall buildings. In more detail, the damage at level 0 (considered in different degrees of severity for D01D05) is very commonly encountered, due to crushing and the high dead load stress values (Pineda, 2016). Diffuse crack patterns in between openings such as windows are also normal in historical masonry structures, due to the concentration of loads (see e.g. Anzani et al. (2000)) and were thus included in D03-D05.

D06 (and to a less extent D07 and D04) considers damage at the belfry. This can be a particularly vulnerable element since there are large windows that can lead to the failure of the relatively slender portions of masonry between them due to shear forces. D07 and D08 were defined accordingly to the dynamic investigation of the current structure, which showed wall local modes at low frequencies associated with low mechanical resistance in the middle part of the building (between level 1 and 2), due to the poor conditions of the masonry and connections between the walls (Ceravolo et al., 2016). D09 represents a widespread worsening of the whole structure overall, while D10-D12 focus on the possible loss of rigidity of the bell tower-church connections along the two main directions. Figure 2 reports the variations of the natural frequencies as induced by these damaged conditions. Figure 3 shows similarly how the mode shapes are affected, especially for the higher modes. Due to space limitations, only the case for the damage scenario D04 is reported. In the MAC matrix, the general term on the $p$-th row and $q$-th column represents the comparison between the $p$-th mode shape of the current structure with the $q$-th mode shape of the D04 model. The matrix is non-symmetric as $\operatorname{MAC}\left(\widehat{\boldsymbol{\varphi}}_{\boldsymbol{p}}^{\boldsymbol{b}}, \widehat{\boldsymbol{\varphi}}_{\boldsymbol{q}}^{\boldsymbol{D} \mathbf{0 4}}\right) \neq$ $\operatorname{MAC}\left(\widehat{\boldsymbol{\varphi}}_{q}^{\boldsymbol{b}}, \widehat{\boldsymbol{\varphi}}_{p}^{D}{ }^{04}\right)$. As it can be seen on the main diagonal, the first five modes remained more related than the other modes with higher natural frequencies. This was noticed for all the cases where extensive damage has been inserted in the model. This point is essential for this discussion, as large damaged areas make the vibrational modes of the structure too different from their original shape to be accurately captured by the initial arrangement of sensors. All the damage scenarios are visualised in Figure 4.

\section{5 | RESULTS}

The dimension of the problem was set to range from 10 to 62 possible alternatives, to inspect the results for a different number of channels. In the following subsections, the results are reported firstly for 20 sensors, to directly compare the results with the sensor network currently deployed and then for less than 20 sensors; the comparison with the classic methodologies described in Section 1 is also reported. The weights were here set as $w_{i}=1 \forall i$, i.e. all damage scenarios were assumed as equally probable to happen. Figure 5 .a shows the positions and directions of all the candidate acquisition channels considered. Figure 5.b shows the layout of the sensor currently in use; the coordinates of the 20 sensors and correspondence with the closest channel ID is explicated in Table 2 . This current setting can be directly compared to the results reported in the following Subsection 5. As it will be shown in more detail in the next Subsections, a single solution was found on the Pareto front in all considered MOGA optimisation cases. 

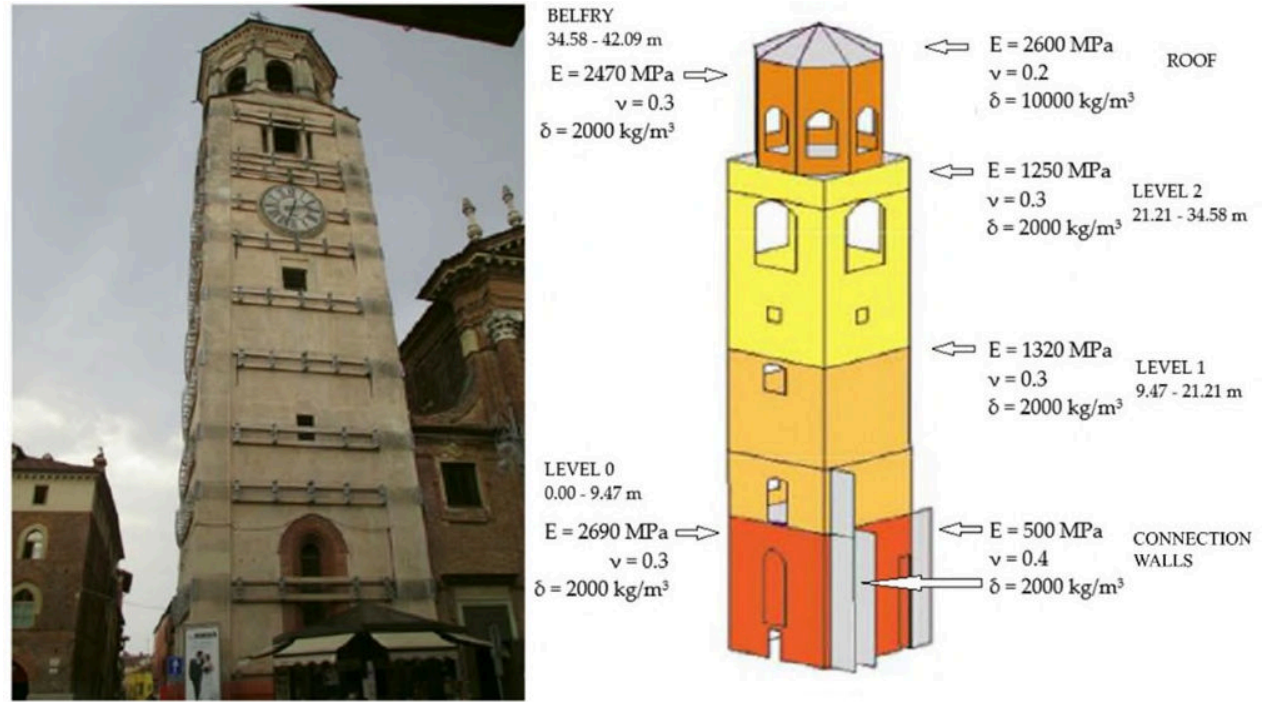

(a)

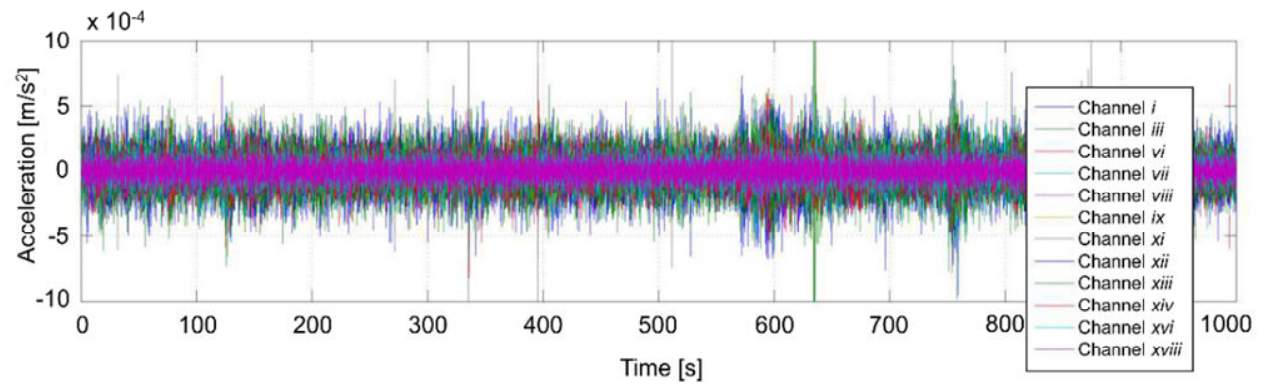

(b)

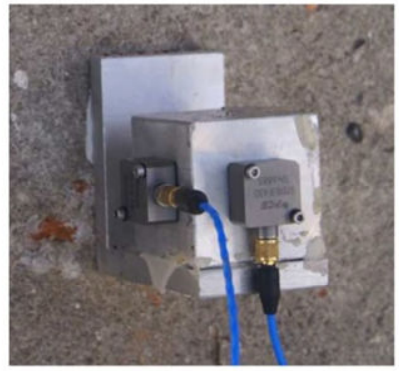

(c)

FIGURE 1. The investigated case study. (a) Left: front view of the bell-tower after the provisional safety measure (2012). Right: FEM with the mechanical properties of the macro-elements after the model updating (adapted from (Ceravolo et al., 2016)). (b) Example of acceleration time history from ambient vibrations (only 12 out of 20 channels displayed). (c) Two uniaxial PCB Piezoeletronics capacitive accelerometers (Model 3701G3FA3G), directed along the $\mathrm{x}$ - and the y-axes, with metal anchorage.

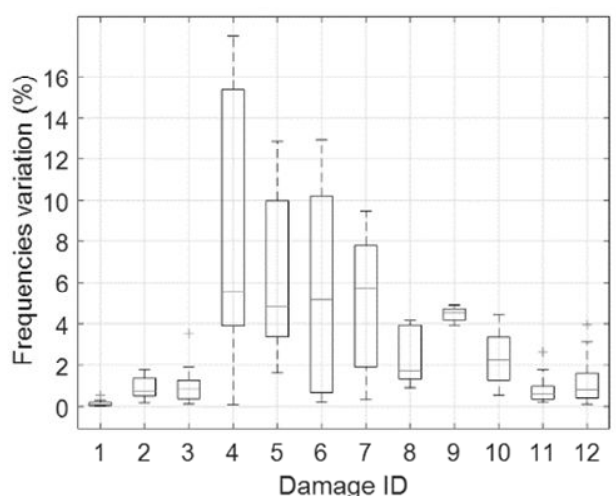

(a)

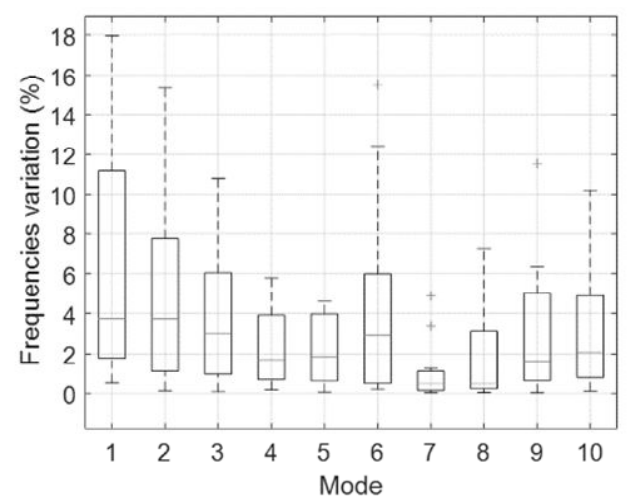

(b)

FIGURE 2. Boxplots of the absolute values of the variation of the natural frequencies: (a) for all modes accordingly to the damage scenario and (b) for all damage scenarios according to the mode number. The central line indicates the median value, the bottom and top edges of the box indicate the 25-th and 75-th percentiles, and the whiskers the largest and smallest data points not considered outliers. The plus symbol indicates the outlier value. 
TABLE 1 Baseline model and other scenarios.

\begin{tabular}{|c|c|c|c|c|c|c|c|c|c|}
\hline 0,99 & 0,01 & 0 & 0,27 & 0,02 & 0,09 & 0 & 0 & 0,18 & 0,04 \\
\hline 0,01 & 0,99 & 0 & 0,01 & 0,25 & 0,05 & 0 & 0,01 & 0,03 & 0,16 \\
\hline 0,02 & 0 & 0,98 & 0 & 0,01 & 0,03 &, 12 & 0,11 & 0 & 0,02 \\
\hline 0,3 & 0,02 & 0 & 0,96 & 0 & 0,3 & 0 & 0,01 & 0,42 & 0,04 \\
\hline 0,04 & 0,29 & 0,01 & 0 & 0,96 & 0, & 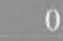 & 0 & 0,01 & 0,42 \\
\hline 0,09 & 0,02 & 0,03 & 0,25 & 0,07 & 0,68 & 0,02 & 0,11 & 0,32 & 0,33 \\
\hline 0,13 & 0 & 0 & 0,18 & 0 & 0,0 & 0,16 & 0,27 & 0,34 & 0,07 \\
\hline 0,21 & 0,02 & 0 & 0,21 & 0 & 0,16 & 0,09 & 0 & 0,66 & 0,02 \\
\hline 0,01 & 0,18 & 0,02 & 0 & 0,16 & 0,07 & 0,14 & 0,17 & 0,06 & 0,45 \\
\hline 0,04 & 0,06 & 0,13 & 0,06 & 0,19 & 0,4 & 0,25 & 0,21 & 0,02 & 0,48 \\
\hline
\end{tabular}

FIGURE 3. Cross-MAC between actual structure and structure with the damage D04.

\begin{tabular}{|c|c|}
\hline Case & Description \\
\hline BASE & As it is, no Young's modulus reductions. \\
\hline D01 & $10.00 \%$ reduction of the $E$ modulus at level 0 , front façade. \\
\hline D02 & $10.00 \%$ reduction of the $E$ modulus at level 0 , all façades. \\
\hline D03 & $\begin{array}{l}10.00 \% \text { reduction of the } E \text { modulus at level } 0 \text {, front façade, } \\
\text { and at level } 1 \text {, front and rear façades between the window } \\
\text { openings. }\end{array}$ \\
\hline D04 & $\begin{array}{l}10.00 \% \text { reduction of the } E \text { modulus at level } 0 \text {, front façade, at } \\
\text { level } 1 \text {, front and rear façades between the window openings, } \\
\text { and at the base of the belfry, all façades. }\end{array}$ \\
\hline D05 & $\begin{array}{l}10.00 \% \text { reduction of the } E \text { modulus at level } 0 \text {, all façades, } \\
\text { and at level } 1 \text {, front and rear façades between the window } \\
\text { openings. }\end{array}$ \\
\hline D06 & $\begin{array}{l}10.00 \% \text { reduction of the } E \text { modulus at the base of the belfry, } \\
\text { all façades. }\end{array}$ \\
\hline D07 & $\begin{array}{l}10.00 \% \text { reduction of the } E \text { modulus at level } 2 \text {, all façades, } \\
\text { and the base of the belfry, all façades. }\end{array}$ \\
\hline D08 & $10.00 \%$ reduction of the $E$ modulus at level 2 , all façades. \\
\hline D09 & $\begin{array}{l}10.00 \% \text { global reduction of the } E \text { modulus of all levels, all } \\
\text { façades }\end{array}$ \\
\hline D10 & $\begin{array}{l}50.0 \% \text { reduction of spring stiffness at the linking with the } \\
\text { Cathedral, } x \text { - and } y \text {-directions }\end{array}$ \\
\hline D11 & $\begin{array}{l}50.0 \% \text { reduction of spring stiffness at the linking with the } \\
\text { Cathedral, } x \text {-direction only }\end{array}$ \\
\hline D12 & $\begin{array}{l}50.0 \% \text { reduction of spring stiffness at the linking with the } \\
\text { Cathedral, } y \text {-direction only }\end{array}$ \\
\hline
\end{tabular}

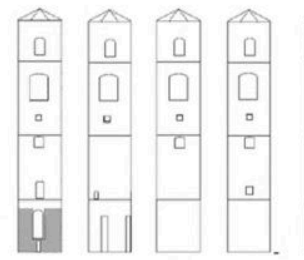

DAMAGE D01-
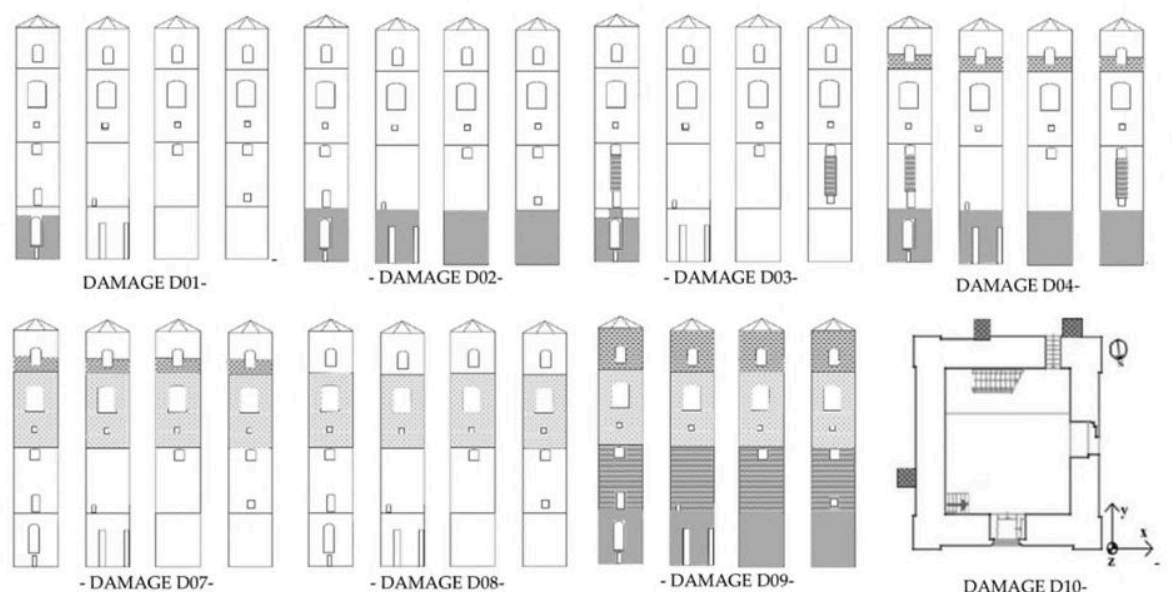

DAMAGE D10-
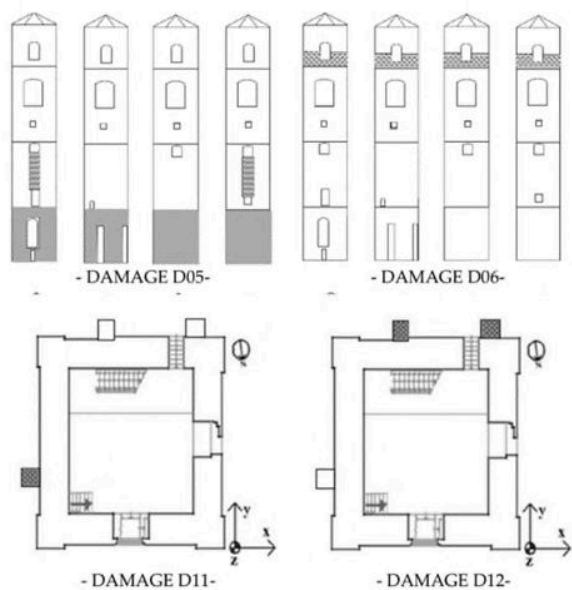

FIGURE 4. The position of the damaged area for the different investigated scenarios. Woung Modulus reduced of $10 \%$ from 2690 $\mathrm{MPa}$ to $2367 \mathrm{MPa}$; $\mathrm{MPa}$ to $2173 \mathrm{MPa}$; Young Modulus reduced of $10 \%$ from $1250 \mathrm{MPa}$ to $1100 \mathrm{MPa}$ Young Modulus reduced of $50 \%$ from 500

$\mathrm{MPa}$ to $250 \mathrm{MPa}$ (connection walls). 


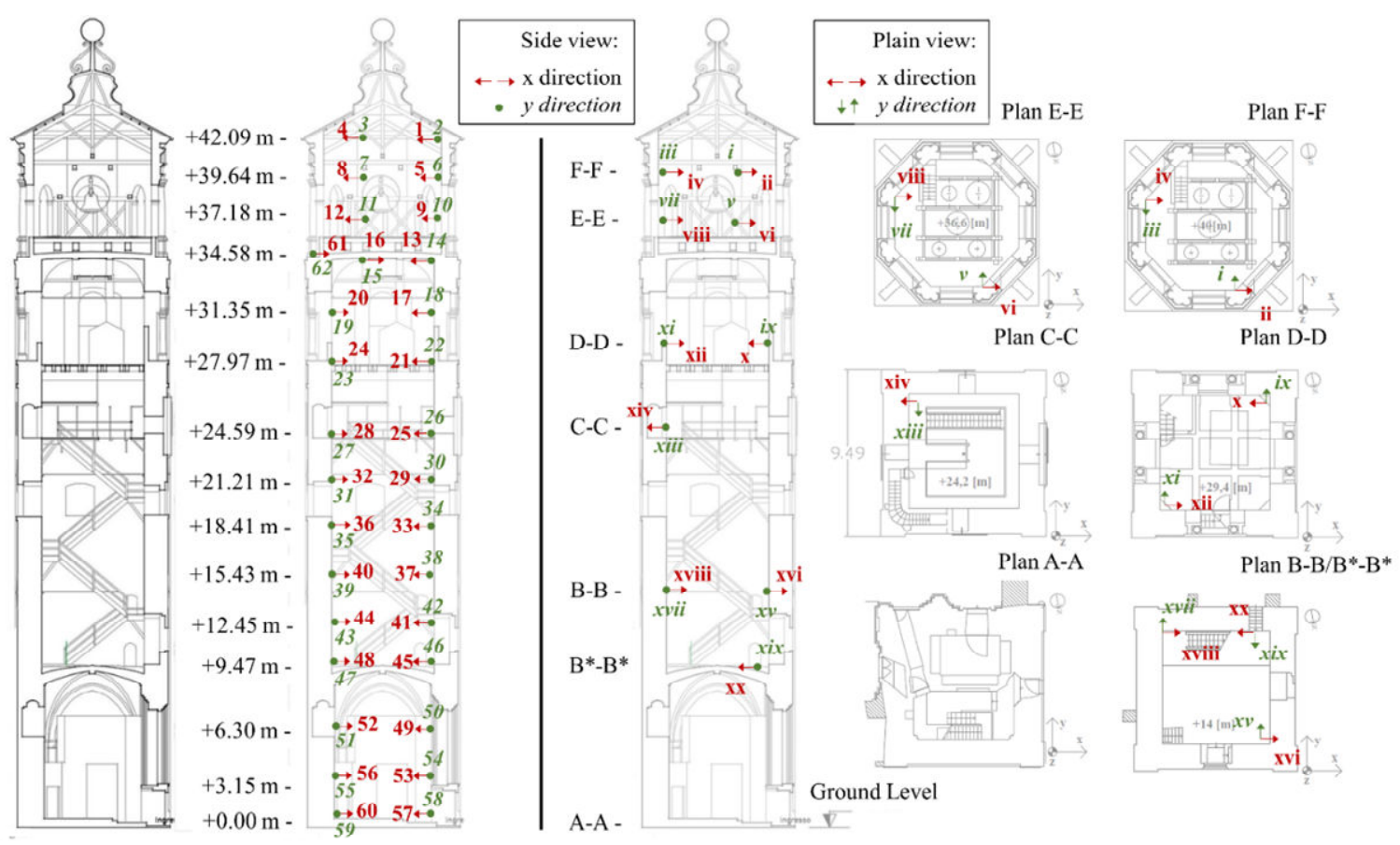

(a)

(b)

FIGURE 5. (a) The bell tower (left) with the location and direction of all potential acquisition channels (right, superimposed to the structure in transparency); (b) current sensor placement (numbered $i$ to $\mathrm{xx}$ ). Channels along y displayed in italic. Channels along $\mathrm{x}$ in plain font.

TABLE 2. Current Sensor Placement and corresponding channel coordinates.

\begin{tabular}{|c|c|c|c|c|c|c|c|c|c|}
\hline $\begin{array}{l}\text { Sensor } \\
\text { ID }\end{array}$ & $\begin{array}{l}\text { corresponding } \\
\text { channel ID \# } \\
\text { (direction) }\end{array}$ & $\begin{array}{c}\mathrm{x} \\
{[\mathrm{m}]}\end{array}$ & $\begin{array}{c}\mathrm{y} \\
{[\mathrm{m}]}\end{array}$ & $\begin{array}{c}\mathrm{z} \\
{[\mathrm{m}]}\end{array}$ & $\begin{array}{l}\text { Sensor } \\
\text { ID }\end{array}$ & $\begin{array}{l}\text { corresponding } \\
\text { channel ID \# } \\
\text { (direction) }\end{array}$ & $\begin{array}{c}\mathrm{x} \\
{[\mathrm{m}]}\end{array}$ & $\begin{array}{c}\mathrm{y} \\
{[\mathrm{m}]}\end{array}$ & $\begin{array}{c}\mathrm{Z} \\
{[\mathrm{m}]}\end{array}$ \\
\hline i & $6(y)$ & 7.08 & 2.09 & 39.64 & xi & $19 *(y)$ & 2.29 & 7.20 & 31.35 \\
\hline ii & $5(x)$ & 7.08 & 2.09 & 39.64 & xii & $20 * *(\mathrm{x})$ & 2.29 & 7.20 & 31.35 \\
\hline iii & $7(y)$ & 2.29 & 7.20 & 39.64 & xiii & $27(y)$ & 2.29 & 7.20 & 24.59 \\
\hline iv & $8(x)$ & 2.29 & 7.20 & 39.64 & xiv & $28(x)$ & 2.29 & 7.20 & 24.59 \\
\hline $\mathrm{v}$ & $10(y)$ & 7.08 & 2.09 & 37.18 & $x v$ & $42(y)$ & 7.08 & 2.09 & 12.45 \\
\hline vi & $9(x)$ & 7.08 & 2.09 & 37.18 & xvi & $41(\mathrm{x})$ & 7.08 & 2.09 & 12.45 \\
\hline vii & $11(\mathrm{y})$ & 2.29 & 7.20 & 37.18 & xvii & $43(y)$ & 2.29 & 7.20 & 12.45 \\
\hline viii & $12(\mathrm{x})$ & 2.29 & 7.20 & 37.18 & xviii & $44(\mathrm{x})$ & 2.29 & 7.20 & 12.45 \\
\hline ix & $18^{*}(\mathrm{y})$ & 7.08 & 2.09 & 31.35 & xix & $46(y)$ & 7.08 & 2.09 & 9.47 \\
\hline$x$ & $17 * *(\mathrm{x})$ & 7.08 & 2.09 & 31.35 & $\mathrm{xx}$ & $45(\mathrm{x})$ & 7.08 & 2.09 & 9.47 \\
\hline
\end{tabular}

\section{1 | Results with 20 Sensors}

Table 3 reports the MOGA results for 20 acquisition channels compared with the OSP obtained from the SO genetic algorithm. The results obtained by applying the non-genetic methods (EI, EVP, ADPR) to the baseline scenario and the damage scenario D03 are also shown for comparison. It can be noticed that these latter sensor patterns are different from what obtained from the same procedures when applied to the baseline model and from what obtained by performing SOGA and MOGA on the baseline and all damaged cases. In particular, EVP and ADPR returned two identical configurations (yet distinct from EI and GA results). When applied to the baseline structure, the selected output channels clustered on the belfry and the top half of the tower. When applied to the damage scenario D03, probably due to the stiffness reduction localised in the bottom half of the tower, the 
sensors were slightly less clustered on the belfry, even if still mostly located in the top half of the structure

Considering the non-genetic options when optimised on the baseline model, only the EI method returned a sensors pattern that produced an adequately low sum of the off-diagonal AutoMAC and Cross-MAC terms if the damage scenarios are taken into account. Indeed, through this method it was still possible to further reduce the number of sensors required while maintaining suitably low off-diagonal values on the AutoMAC; a layout of 14 sensors was obtained (this will be better discussed in the next subsection). EVP and ADPR returned higher off-diagonal AutoMAC terms. Only with many more channels (about 30) they reached a goodness-of-fit comparable with EI results. By way of illustration, Table 4 details the sum of the diagonal and of the off-diagonal terms obtained by fitting the EI, SOGA, and MOGA algorithms on the damage scenarios D03 and D04. As expected, for the EI algorithm, the sensor patterns optimised over the baseline structure struggle to recognise the mode shapes of the damaged cases, especially D04, and vice versa. SOGA and MOGA performed comparably in this study. It must be remembered that the optimisation task was set to minimise the off-diagonal elements of the AutoMAC and to minimise both the off-diagonal and the diagonal terms of the several Cross-MAC matrices. As it can be inferred, the GAs fit better the different damage scenarios, resulting in a lower sum of both the diagonal and the offdiagonal terms in Cross-MAC matrices and thus highlighting the differences between the damaged and the baseline mode shapes, as required for damage detection. Regarding the AutoMAC, the results are still comparable to the ones of the EI method fitted on the current model. Noteworthy, the diagonal terms of the Cross-MAC matrices obtained by fitting the EI algorithm on the damage scenarios are lower than their counterparts from genetic algorithms. However, their offdiagonal elements are much higher. That indicates that this lowering is not produced by a better discerning of the damaged mode shapes respect to their corresponding baseline ones, but rather by an overall inefficiency of the arrangement to distinguish both damaged or baseline eigenshapes. Hence, it is important to stress that both the off-diagonal and the diagonal terms of the Cross-MAC matrices must be minimised simultaneously.

\section{2 | Results with Less than 20 Sensors}

The techniques applied showed that it was possible to reduce the number of sensors without losing performance (in terms of off-diagonal error) with less than the 20-channels configuration currently deployed. Specifically, both the EI technique and SOGA still outperformed the current configuration with as low as 14 channels for the baseline scenario. These resulting arrangements are reported in Table 5 and compared with
MOGA-produced OSP with the same number of sensors. In the arrangement found employing EI, all the 14 sensors are clustered together in a delimited area of the bell tower, with the risk of multiple sensor failures in case of damage.

\section{3 | Results Considering the Likelihood of the Potential Damage Scenarios}

Here, different values of the weights have been assigned. By zeroing some of them it is possible to focus on a subset of the 12 cases reported in Table 1, thus investigating the importance of adding or neglecting a potential damage scenario. On the other hand, considering $0<w<1$ can be used to reflect the different likelihood of the several cases, or to model any other designer's choice. For $w=\left[w_{0}, w_{1}, w_{2}, \ldots, w_{M}\right]$, considering $w_{0}$ related to the baseline model, four cases have been analysed for both MOGA and single-OF GA:

$$
\begin{aligned}
& w_{0}=w_{1}=1, w_{k}=0 \forall k \neq 0,1 ; \\
& w_{0}=w_{4}=1, w_{k}=0 \forall k \neq 4 \\
& \left\{\begin{array}{c}
w_{k}=1 \text { if } k=0,1,3,4 \\
w_{k}=0 \text { otherwise }
\end{array}\right. \\
& w=[1.00,0.92,0.75,1.00, \ldots \\
& \ldots, 0.83,0.67,0.17,0.50,0.58,0.08,0.42,0.25,0.34]
\end{aligned}
$$

Case I focuses on the simple damage scenario D01, with only one external façade cracked at the tower bell bases. As explained before, this is very common in this kind of structures. It was also considered that the investigated bell tower is relatively higher than similar structures found elsewhere in northern Italy. This makes the development of cracks at the base and lower levels more probable. Case II considers again a unique damage case, D04, but with the cracked masonry more spread throughout the whole length of the structure. Case III includes a subset of three damage scenarios considered very likely to happen and discard the other ones. Finally, Case IV exemplifies a potential ranking of likelihoods, with the damage scenario deemed as the most probable one, D03, taken at full weight (1) and the other ones in descending order (with constant steps equal to $1 / 12$ ). The baseline configuration is assumed at full weight in all cases. Any other arrangement of the weights can be applied, according to the preferences of the designer; the ones reported here serve as an example of this potentiality.

Table 7 reports the resulting OSP for cases (I)-(IV) for both SOGA and MOGA, with 20 and 14 sensors. The results in Table 7 can be compared to the ones in Table 3 and Table 5. It can be noticed that the resulting sensor placements are generally different, particularly for the MOGA, even if the sensors tend to cluster in the same areas; on the other hand, the SOGA method returned the same sensor placement for the Case 
(I) with 14 sensors. In Table 6 the diagonal and off-diagonal terms for the MAC matrices corresponding to cases (I)-(IV) are reported, considering 20 acquisition channels. The results follow the same behaviour previously described in Table 4 . Case (I) struggles to recognise the mode shapes belonging to the fourth damage scenario, resulting in low diagonal terms but high off-diagonal elements in the BASE -D04 Cross-MAC. Case (II) suffers even more for both the Cross-MAC matrices BASE -D01 and BASE -D03. Only Cases (III) and (IV) produce results comparable to the ones of Table 4 (remembering that even in the best case the sensor placement was never totally able to recognise the higher modes of D04, which deviate the most from the intact structure). These findings point out how the optimisation provides reliable results only for the selected damage scenarios or from cases which do not deviate extremely from the ones included (e.g. Case (I) still performed relatively well for D03 without being optimised for it). The MOGA has lower diagonal values on the Cross-MAC cases, i.e. it distinguishes better the damaged mode shapes from their baseline counterparts. For illustrative purposes, some results are reported in Figure 6; the sensor placement changes noticeably with respect to the classic methods (e.g. EVP), while the GAs generally return more uniformly spread sensor networks.

TABLE 3. Resulting OSP with 20 sensors.

\begin{tabular}{|c|c|c|}
\hline \multicolumn{3}{|c|}{ channel ID \# } \\
\hline & Channels along the $\mathrm{x}$-direction & Channels along the y-direction \\
\hline MOGA $^{1}$ & $9-13-16-20-21-24-28-37-44-45-53$ & $10-19-23-27-31-38-39-46-47$ \\
\hline SOGA $^{1}$ & $5-8-12-20-21-24-28-33-41-48-49-52-53$ & $11-14-15-27-43-46-47$ \\
\hline $\mathrm{EI}^{2}$ & $1-4-12-20-21-28-29-33-36-44$ & $2-3-14-18-19-23-26-27-34-35$ \\
\hline $\mathrm{EVP}^{2}$ & $1-4-5-8-12-16-17-20-24-28$ & $2-3-6-7-10-11-18-23-26-31$ \\
\hline $\mathrm{ADPR}^{2}$ & $1-4-5-8-12-16-17-20-24-28$ & $2-3-6-7-10-11-18-23-26-31$ \\
\hline $\mathrm{EI}^{3}$ & $1-4-16-20-21-28-33-36-40-44$ & $2-3-18-19-26-31-34-35-39-43$ \\
\hline $\mathrm{EVP}^{3}$ & $1-4-5-12-20-24-28-36$ & $2-3-6-10-11-18-23-26-27-31-35-39$ \\
\hline $\mathrm{ADPR}^{3}$ & $1-4-5-12-20-24-28-36$ & $2-3-6-10-11-18-23-26-27-31-35-39$ \\
\hline
\end{tabular}

${ }^{1}$ optimised on all scenarios ('as it is' and damaged ones).

2 applied to the current structural situation.

${ }^{3}$ applied to the damaged scenario D03

TABLE 4. Diagonal and off-diagonal terms for the MAC matrices.

\begin{tabular}{cccccc}
\hline $\begin{array}{c}\text { Sum of diagonal terms } \\
\begin{array}{c}\text { Sum of } \\
\text { off-diagonal terms }\end{array}\end{array}$ & $\begin{array}{c}\text { EI algorithm } \\
\text { (optimised } \\
\text { on BASE ) }\end{array}$ & $\begin{array}{c}\text { EI algorithm } \\
\text { (optimised } \\
\text { on D03) }\end{array}$ & $\begin{array}{c}\text { EI algorithm } \\
\text { (optimised } \\
\text { on D04) }\end{array}$ & MOGA & SOGA \\
\hline AutoMAC BASE & 10.00 & 9.71 & 6.37 & 10.00 & 10.00 \\
& 13.38 & 10.52 & 10.70 & 4.08 & 4.26 \\
Cross-MACBASE - D03 & 9.72 & 10.00 & 6.12 & 9.64 & 9.69 \\
Cross-MAC BASE - D04 & 13.94 & 11.21 & 11.3 & 4.39 & 4.46 \\
& 6.72 & 6.08 & 10.00 & 6.16 & 6.44 \\
\hline
\end{tabular}

TABLE 5 Resulting OSP with 14 sensors.

\begin{tabular}{ccc}
\hline \multicolumn{3}{c}{ channel ID \# } \\
\hline & Channels along the x-direction & Channels along the y-direction \\
\hline MOGA $^{1}$ & $9-17-20-29-36-37-44-45-48$ & $10-14-15-23-39$ \\
SOGA $^{1}$ & $17-20-25-37-49$ & $7-10-14-15-22-31-39-46-51$ \\
EI $^{2}$ & $1-4-17-28-29-33-36$ & $18-19-23-26-27-34-35$ \\
$\mathrm{EI}^{3}$ & $1-4-16-29-33-44$ & $18-19-26-27-34-35-38-39$ \\
\hline
\end{tabular}

${ }^{1}$ optimised on all scenarios ('as it is' and damaged ones).

2 applied to the current structural situation.

3 applied to the damaged scenario D03 
TABLE 6. Diagonal and off-diagonal terms for the MAC matrices (cases I-IV, with 20 channels).

\begin{tabular}{ccccc|cccc}
\hline & \multicolumn{3}{c}{ MOGA } & \multicolumn{5}{c}{ SOGA } \\
\cline { 2 - 8 } & I & II & III & IV & I & II & III & IV \\
\hline AutoMAC & 10.00 & 10.00 & 10.00 & 10.00 & 10.00 & 10.00 & 10.00 & 10.00 \\
BASE & 4.10 & 4.28 & 4.18 & 4.60 & 4.25 & 4.71 & 4.28 & 4.91 \\
Cross-MAC & 9.99 & 6.14 & 9.81 & 9.9 & 10.00 & 9.99 & 9.99 & 9.97 \\
BASE - D01 & 4.11 & 6.81 & 4.29 & 4.314 & 4.20 & 4.69 & 4.30 & 4.49 \\
Cross-MAC & 9.31 & 6.03 & 9.69 & 9.71 & 8.67 & 9.66 & 9.77 & 9.71 \\
BASE - D03 & 4.67 & 6.43 & 4.63 & 4.67 & 5.43 & 4.70 & 4.06 & 4.04 \\
Cross-MAC & 6.07 & 8.09 & 6.77 & 6.56 & 6.15 & 8.65 & 6.94 & 7.11 \\
BASE - D04 & 6.67 & 4.35 & 6.16 & 4.63 & 6.72 & 6.95 & 5.54 & 5.44 \\
\hline
\end{tabular}

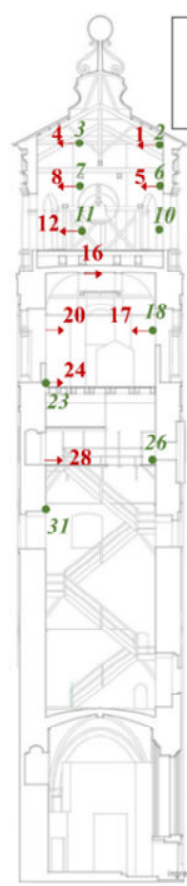

(a)

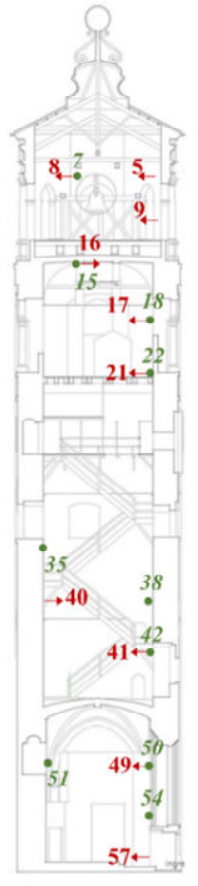

(c)

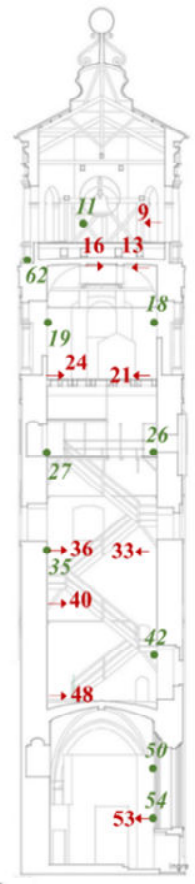

(d)

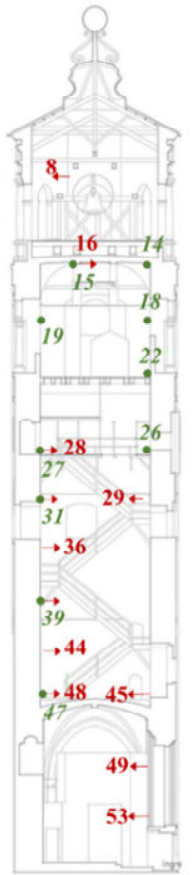

(e)

FIGURE 6. Some illustrative example of sensor patterns with 20 channels. (a) EVP optimised on the undamaged scenario (very clustered to the top); (b) EVP optimised on D03; (c) and (d) MOGA and SOGA optimised on all cases (baseline and damaged); (e) MOGA optimised on the baseline, D01, D03, and D04 (Case III) 
TABLE 7. Resulting OSP with weighted damage scenarios.

channel ID \#

\begin{tabular}{|c|c|c|c|}
\hline \multicolumn{2}{|c|}{$\begin{array}{l}\text { With } 20 \\
\text { sensors }\end{array}$} & Channels along the $\mathrm{x}$-direction & Channels along the y-direction \\
\hline \multirow{2}{*}{ (I) } & MOGA & $5-8-9-16-17-21-40-41-49-57$ & $7-15-18-22-35-38-42-50-51-54$ \\
\hline & SOGA & $9-13-16-21-24-33-36-40-48-53$ & $11-18-19-26-27-35-42-50-54-62$ \\
\hline \multirow{2}{*}{ (II) } & MOGA & $12-13-17-20-25-36-37-41-44-48-49$ & $14-18-22-23-30-31-38-46-55$ \\
\hline & SOGA & $5-9-13-20-21-29-37-40-48-52-56-60$ & $7-11-35-38-43-46-51-55$ \\
\hline \multirow{2}{*}{ (III) } & MOGA & $8-16-28-29-36-44-45-48-49-53$ & $14-15-18-19-22-26-27-31-39-47$ \\
\hline & SOGA & $12-13-17-20-24-29-33-40-48-49-52$ & $18-19-30-38-39-42-43-54-55$ \\
\hline \multirow{2}{*}{ (IV) } & MOGA & $8-13-20-21-29-37-45-48-49$ & $14-15-23-26-30-31-34-38-39-43-46$ \\
\hline & SOGA & $5-8-12-16-24-28-37-45-53-56$ & $15-18-19-26-30-38-42-43-50-51$ \\
\hline \multicolumn{2}{|c|}{$\begin{array}{l}\text { With } 14 \\
\text { sensors }\end{array}$} & Channels along the $\mathrm{x}$-direction & Channels along the y-direction \\
\hline \multirow{2}{*}{ (I) } & MOGA & $12-17-20-28-33-40-52-53$ & $14-19-22-26-27-39$ \\
\hline & SOGA & $17-20-25-37-49$ & $7-10-14-15-22-31-39-46-51$ \\
\hline \multirow{2}{*}{ (II) } & MOGA & $5-9-21-28-37-40-56-60$ & $10-14-18-19-46-47$ \\
\hline & SOGA & $5-8-16-44-45-57-60-61$ & $11-19-22-47-51-54$ \\
\hline \multirow{2}{*}{ (III) } & MOGA & $9-16-24-29-37-44-45-52$ & $15-22-30-38-51-55$ \\
\hline & SOGA & $5-9-16-20-21-37-52$ & $10-38-39-43-46-51-62$ \\
\hline \multirow{2}{*}{ (IV) } & MOGA & $17-20-29-36-37-40-48-60$ & $6-7-14-19-43-47-$ \\
\hline & SOGA & $13-16-21-44-57-60-61$ & $7-10-23-31-39-51-55$ \\
\hline
\end{tabular}

\section{4 | Selected Optimal Sensor Placement}

By applying the MOGA approach, it was found that a setup with 16 channels (for which a unique solution exists) provided good results, with $f_{1}(\boldsymbol{x})=0.04$ and $f_{2}(\boldsymbol{x})=0.27$ (considering all the weights equal to 1 ). These were the best results compared to any other sensor pattern with the same number of channels. By further reducing the number of sensors deployed, it was observed that both cost functions grew higher (especially $f_{1}(\boldsymbol{x})$ became larger than $5 \%$ ), making the candidate sensor patterns too imprecise. Several other practical issues, such as the easiness of accessibility, the requirement of redundancy in case of sensor failure, and a preference to install biaxial accelerometers at the same location rather than more uniaxial accelerometers at distinct yet nearby positions, were considered when validating this choice. In detail, the proposed network is composed of 2 biaxial and 12 monoaxial accelerometers, 9 oriented along the $\mathrm{x}$-axis and 7 along the $\mathrm{y}$ axis. The selected channels and corresponding coordinates are enlisted in Table 8. The sensor network is portrayed in Figure 7. By comparing this solution with the current layout (Figure 5.b) it is evident how the sensors are much less clustered at the tower top and more uniformly spread along the whole tower.

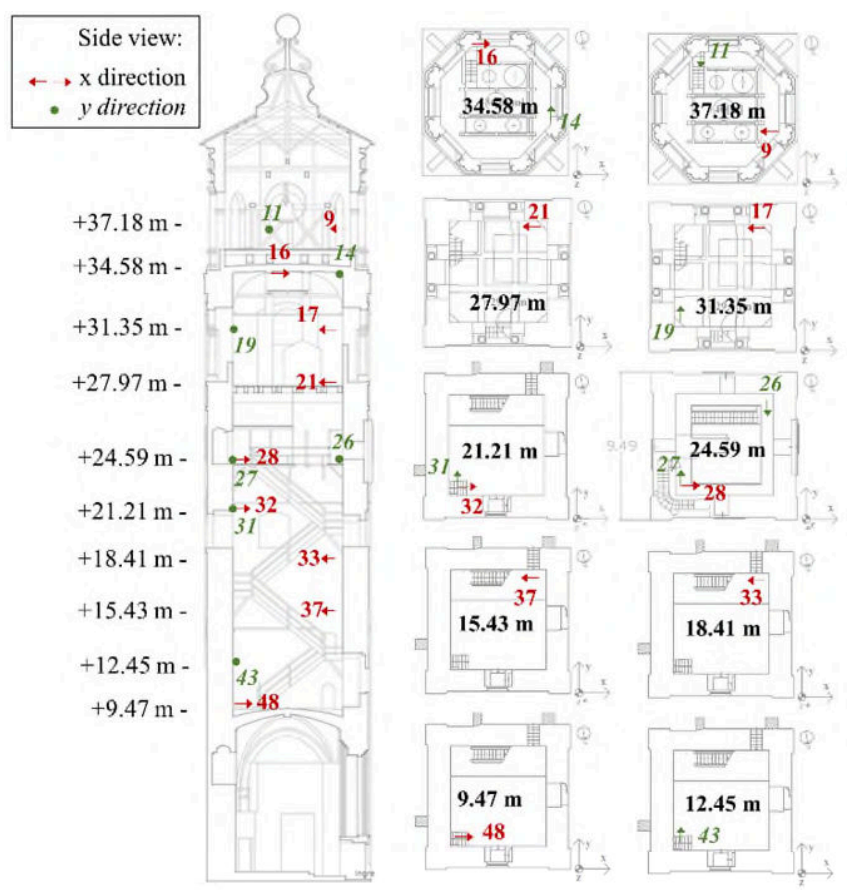

FIGURE 7. Resulting OSP with 16 sensors (MOGA approach). 
TABLE 8. Optimal Sensor Placement (MOGA approach, 16 sensors).

\begin{tabular}{|c|c|c|c|c|}
\hline $\begin{array}{c}\text { Sensor } \\
\text { ID \# }\end{array}$ & $\begin{array}{c}\text { channel ID } \\
\# \\
\text { (direction) }\end{array}$ & $x[\mathrm{~m}]$ & $\mathrm{y}[\mathrm{m}]$ & $\begin{array}{c}\mathrm{z} \\
{[\mathrm{m}]}\end{array}$ \\
\hline 1 & $9(\mathrm{x})$ & 7.08 & 2.09 & 37.18 \\
\hline 2 & $11(\mathrm{y})$ & 2.29 & 7.20 & 37.18 \\
\hline 3 & $14(\mathrm{y})$ & 7.08 & 2.09 & 34.58 \\
\hline 4 & $16(x)$ & 2.29 & 7.20 & 34.58 \\
\hline 5 & $17(\mathrm{x})$ & 7.08 & 2.09 & 31.35 \\
\hline 6 & $19(\mathrm{y})$ & 2.29 & 7.20 & 31.35 \\
\hline 7 & $21(\mathrm{x})$ & 7.08 & 2.09 & 27.97 \\
\hline 8 & $26(y)$ & 7.08 & 2.09 & 24.59 \\
\hline 9 & $27(\mathrm{y})$ & 2.29 & 7.20 & 24.59 \\
\hline 10 & $28(x)$ & 2.29 & 7.20 & 24.59 \\
\hline 11 & $31(\mathrm{y})$ & 2.29 & 7.20 & 21.21 \\
\hline 12 & $32(x)$ & 2.29 & 7.20 & 21.21 \\
\hline 13 & $33(x)$ & 7.08 & 2.09 & 18.41 \\
\hline 14 & $37(x)$ & 7.08 & 2.09 & 15.43 \\
\hline 15 & $43(\mathrm{y})$ & 2.29 & 7.20 & 12.45 \\
\hline 16 & $48(x)$ & 2.29 & 7.20 & 9.47 \\
\hline
\end{tabular}

\section{5 | Final Discussion}

The MOGA approach has been benchmarked against the currently deployed sensor network, a single-OF GA approach, and three classic (non-GA-based) methodologies, specifically:

- ADPR and EVP have been applied to the baseline model, considering 20 acquisition channels; they were both outperformed by the other genetic and nongenetic alternatives.

- EI has been applied to the baseline and damaged models, considering 20 or fewer channels and providing better results than ADPR and EVP.

- Single-OF (classic) GA and MOGA have been applied to the baseline and damaged models, considering the whole set of the damage scenarios or a subgroup of it, for 20 or fewer channels.

The MOGA results were comparable to the ones of EI and classic GA for the AutoMAC of the current structural condition and the single damage scenarios and more robust of the other options for the Cross-MAC on the several baseline-damaged combinations. The MOGA function returned a unique solution for all the damages considered. This seems to indicate that, for these specific damage scenarios and application, the two OFs are so closely correlated that the Pareto front of the equivalent solutions is reduced to a 0 -dimensional point. This can be most probably explained by the resemblance between the pre- and the post-damage mode shapes. Damage severity plays an important role in this, but the damage pattern is also very relevant.

\section{6 | CONCLUSIONS}

The performance and the reliability of an SHM system are related to the capability of the sensors to identify any deviation from a baseline model. Extensive damage can alter the structure of interest, causing an OSP suited for the initial structural conditions to eventually become less, or not at all, effective. To overcome this issue, a Multi-Objective GA approach has been proposed in this paper, including both information from the current structure and some potential damage scenarios. The aim was to define a pre-emptive arrangement of the sensors, able to preserve its functionality in case of damage-related changes in the structure of the target building. The problem is multiobjective, as the aim is to optimise the sensor network over different structural configurations and to make the mode shapes of the damaged structure clearly recognisable from their equivalent of the baseline model. The calibrated FE model of the bell tower of the Santa Maria and San Giovenale Cathedral in Fossano (Piedmont, Italy), was investigated as a case study.

The major limitation of the proposed approach is that the potential damage scenarios have to be envisaged a priori, thus the method requires a certain knowledge of the most likely damage scenarios. Nevertheless, by combining the historical records of surveys taken from similar structure damaged after seismic events and extensive testing of the target structure, some educated guesses can be made. Relatively simple structures such as bell towers generally undergo some very specific damage patterns, which are frequently encountered. Damage may nevertheless occur in a non-foreseeable way, with losses of material stiffness in unexpected locations. Yet, the proposed approach still guarantees a certain degree of robustness in case of seismic events, much larger than for standard procedures, where potential damage-induced structural changes are completely disregarded from the OSP problem. Rearranging the sensor network as proposed will not cause any worsening of the performance on the current case, while it has more probability of remaining efficient in the case of future seismic damage, which is plausible in the seismically active area.

The work presented here leaves large room for improvements. The economic and practical considerations not related to damage have not been considered. For instance, channels with different orientations but sharing the same position would be preferred rather than distinct locations, as it is more convenient to install one tri- or bi-axial accelerometers rather than two or more uniaxial sensors. On the other hand, less clustered sensors would be preferable in case of local structural 
failures. Locations that are more difficult to access may be marked by a penalty function as well. All these considerations can be fit in a more complicated, larger-dimensional multioptimisation task. Finally, the two OFs were assumed here as equally important. However, it may be acceptable to consider a loss in one aspect, if this brings improvement in another one which is considered more important. All these aspects will be analysed in future works.

\section{REFERENCES}

Adeli, H., \& Cheng, N. T. (1994a). Augmented Lagrangian genetic algorithm for structural optimization. Journal of Aerospace Engineering, 7(1), 104-118.

Adeli, H., \& Cheng, N. T. (1994b). Concurrent genetic algorithms for optimization of large structures. Journal of Aerospace Engineering, 7(3), 276-296.

Adeli, H., \& Kumar, S. (1995a). Distributed genetic algorithm for structural optimization. Journal of Aerospace Engineering, 8(3), 156-163.

Adeli, H., \& Kumar, S. (1995b). Concurrent structural optimization on massively parallel supercomputer. Journal of Structural Engineering, 121(11), 1588-1597.

Adeli, H., \& Sarma, K. C. (2006). Cost optimization of structures: fuzzy logic, genetic algorithms, and parallel computing. John Wiley \& Sons.

Allemang, R. J. (2003). The modal assurance criterion-twenty years of use and abuse. Sound and vibration, 37(8), 14-23.

Anzani, A. N. N. A., Binda, L., \& Roberti, G. M. (2000). The effect of heavy persistent actions into the behaviour of ancient masonry. Materials and Structures, 33(4), 251-261. https://doi.org/10.1016/j.engfailanal.2016.02.013

Antunes, C. H., Alves, M. J., \& Clímaco, J. (2016). Multiobjective Linear and Integer Programming. Springer International Publishing, Cham. https://doi.org/10.1007/978-3-319-28746-1.

Barontini, A., Masciotta, M. G., Ramos, L. F., Amado-Mendes, P., \& Lourenço, P. B. (2017). An overview on natureinspired optimization algorithms for Structural Health Monitoring of historical buildings. Procedia engineering, 199 , https://doi.org/10.1016/j.proeng.2017.09.439

Binh, T. T., \& Korn, U. (1997). MOBES: A multiobjective evolution strategy for constrained optimization problems. In The third international conference on genetic algorithms 25, 176-182.

Bursi, O. S., \& Wagg, D. (2009). Modern testing techniques for structural systems: dynamics and control, 502. Springer Science \& Business Media.

Censor, Y. (1977). Pareto optimality in multiobjective problems. Applied Mathematics and Optimization, 4(1), 4159. https://doi.org/10.1007/BF01442131.

Ceravolo, R., \& Abbiati, G. (2013). Time domain identification of structures: comparative analysis of output-only methods. Journal of Engineering Mechanics, 139(4), 537-544.

Ceravolo, R., Pistone, G., Fragonara, L. Z., Massetto, S., \& Abbiati, G. (2016). Vibration-based monitoring and diagnosis of cultural heritage: a methodological discussion in three examples. International Journal of Architectural Heritage, 10(4), 375-395. https://doi.org/10.1080/15583058.2013.850554

Chang, M., Pakzad, S..N..(2014). Optimal sensor placement for modal identification of bridge systems considering number of sensing nodes. Journal of Bridge Engineering 19(6),04014019 https://doi.org/10.1061/(ASCE)BE.19435592.0000594

Chiorino, M. A., Ceravolo, R., Spadafora, A., Zanotti Fragonara, L., \& Abbiati, G. (2011). Dynamic characterization of complex masonry structures: the Sanctuary of Vicoforte. International Journal of Architectural Heritage, 5(3), 296-314. https://doi.org/10.1080/15583050903582516.

Civera, M., Ferraris, M., Ceravolo, R., Surace, C., \& Betti, R. (2019). The Teager-Kaiser Energy Cepstral Coefficients as an Effective Structural Health Monitoring Tool. Applied Sciences, 9(23), 5064. https://doi.org/10.3390/app9235064.

Ciro, G. C., ., Dugardin, F., Yalaoui, F., \& Kelly, R. (2016). A NSGA-II and NSGA-III comparison for solving an open shop scheduling problem with resource constraints. IFACPapersOnLine, $\quad$ 49(12),

1272-1277. https://doi.org/10.1016/j.ifacol.2016.07.690

Deb, K., Pratap A., Agarwal S., Meyarivan T. (2002). A fast and elitist multiobjective genetic algorithm: NSGA-II. IEEE transactions on evolutionary computation, 6(2), 182-197. https://doi.org/10.1109/4235.996017

Doebling, S., Peterson, L., \& Alvin, K. (1995). Measurement of static flexibility matrices for experiments with incomplete reciprocity. In 36th Structures, Structural Dynamics and Materials Conference, pp. 2777-2791. https://doi.org/10.2514/6.1995-1092.

Ferraris, M., Civera, M., Ceravolo, R., Surace, C., \& Betti, R. (2020). Using enhanced cepstral analysis for structural health monitoring. In Proceedings of the 13th International Conference on Damage Assessment of Structures, pp. 150165. https://doi.org/10.1007/978-981-13-8331-1_11.

Goldberg, D. E. (1989). Genetic algorithms in search. Optimization, and MachineLearning. Addison-Wesley Longman Publishing Co., Inc., 1989. (accessed February 28, 2019). https://dl.acm.org/citation.cfm/id=534133

Gutierrez Soto, M. and Adeli, H. (2013), Placement of Control Devices for Passive, Semi-active, and Active, Vibration Control of Structures, Scientia Iranica - Transaction A: Civil Engineering, 20(6), pp. 1567-1578

Hasançebi, O., \& Erbatur, F. (2000). Evaluation of crossover techniques in genetic algorithm based optimum structural design. Computers \& Structures, 78(1-3), 435-448. https://doi.org/10.1016/S0045-7949(00)00089-4

Ishibuchi, H., Tsukamoto, N., \& Nojima, Y. (2008, June). Evolutionary many-objective optimization: A short review. In 2008 IEEE World Congress on Computational Intelligence pp. 2419-2426. 10.1109/CEC.2008.4631121

Kammer, D. C. (1991). Sensor placement for on-orbit modal identification and correlation of large space structures. Journal of Guidance, Control, and Dynamics, 14(2), 251-259. https://doi.org/10.2514/3.20635.

Lenticchia, E., Ceravolo, R., \& Antonaci, P. (2018). Sensor 
placement strategies for the seismic monitoring of complex vaulted structures of the modern architectural heritage. Shock and Vibration, 2018. https://doi.org/10.1155/2018/3739690

Lenticchia, E., Ceravolo, R., \& Chiorino, C. (2017). Damage scenario-driven strategies for the seismic monitoring of XX century spatial structures with application to Pier Luigi Nervi's Turin Exhibition Centre. Engineering Structures, 137, 256-267. https://doi.org/10.1016/j.engstruct.2017.01.067.

Li, D. S., Li, H. N., \& Fritzen, C. P. (2007). The connection between effective independence and modal kinetic energy methods for sensor placement. Journal of sound and vibration, 305(4-5),

945-955. https://doi.org/10.1016/j.jsv.2007.05.004.

Martucci, D., Civera, M., Surace, C., \& Worden, K. (2018, October). Novelty detection in a cantilever beam using extreme function theory. In Journal of Physics: Conference Series, 1106(1), IOP Publishing. 012027. https://doi.org/10.1088/1742-6596/1106/1/012027.

Munk, D. J., Gareth A. V., \& Grant P. S. (2015). Topology and shape optimization methods using evolutionary algorithms: a review. Structural and Multidisciplinary Optimization,52, 613-631. https://doi.org/10.1007/s00158-015-1261-9.

Papadimitriou, C., Beck, J.L., Au, S.K. (2000). Entropy-based optimal sensor location for structural model updating. Journal of Vibration and Control 6(5), 781-800. https://doi.org/10.1177/107754630000600508

Penny, J. E. T., Friswell, M. I., \& Garvey, S. D. (1994). Automatic choice of measurement locations for dynamic testing. AIAA journal, 32(2), 407-414. https://doi.org/10.2514/3.11998.

Pineda, P. (2016). Collapse and upgrading mechanisms associated to the structural materials of a deteriorated masonry tower. Nonlinear assessment under different damage and loading levels. Engineering Failure Analysis, 63, 72-93. https://doi.org/10.1016/j.engfailanal.2016.02.013

Qin, A. K., \& Suganthan, P. N. (2005). Self-adaptive differential evolution algorithm for numerical optimization. 2005 IEEE congress on evolutionary computation, 1785-1791

Rafiei, M.H. and Adeli, H. (2017). A Novel Machine Learning Based Algorithm to Detect Damage in Highrise Building Structures, The Structural Design of Tall and Special Buildings, 26(18), DOI: 10.1002/tal.1400

Raich, A. M., \& Liszkai, T. R. (2012). Multi-objective optimization of sensor and excitation layouts for frequency response function-based structural damage identification. Computer-Aided Civil and Infrastructure Engineering, 27(2), 95-117.

Rudenko, O., \& Schoenauer, M. (2004). A steady performance stopping criterion for Pareto-based evolutionary algorithms. In 6th International Multi-Objective Programming and Goal Programming Conference.

Saleh, A., \& Adeli, H. (1994a). Parallel algorithms for integrated structural/control optimization. Journal of Aerospace Engineering, 7(3), 297-314.

Saleh, A., \& Adeli, H. (1994b). Microtasking, macrotasking, and autotasking for structural optimization. Journal of Aerospace Engineering, 7(2), 156-174.
Sarma, K. C., \& Adeli, H. (2000a). Fuzzy genetic algorithm for optimization of steel structures. Journal of Structural Engineering, 126(5), 596-604.

Sarma, K. C., \& Adeli, H. (2000b). Fuzzy discrete multicriteria cost optimization of steel structures. Journal of structural engineering, 126(11), 1339-1347.

Schaffer, J. D. (1985). Multiple objective optimization with vector evaluated genetic algorithms. In Proceedings of the first international conference on genetic algorithms and their applications, 1985.

Serpik, I. N., Alekseytsev, A. V., \& Balabin, P. Y. (2017). Mixed approaches to handle limitations and execute mutation in the genetic algorithm for truss size, shape and topology optimization. Periodica Polytechnica Civil Engineering, 61(3), 471-482. https://doi.org/10.3311/PPci.8125

Srinivas, N., \& Deb, K. (1994). Muiltiobjective optimization using nondominated sorting in genetic algorithms. Evolutionary computation, 2(3), 221-248.

Sun, H., \& Betti, R. (2015). A hybrid optimization algorithm with Bayesian inference for probabilistic model updating. Computer-Aided Civil and Infrastructure Engineering, 30(8), 602-619.

2004-2012. https://doi.org/10.1016/j.compstruc.2008.05.001.

Tondini, N., Bursi, O. S., Bonelli, A., \& Fassin, M. (2015). Capabilities of a fiber bragg grating sensor system to monitor the inelastic response of concrete sections in new tunnel linings subjected to earthquake loading. Computer-Aided Civil and Infrastructure Engineering, 30(8), 636-653.

Tong, K. H., Bakhary, N., Kueh, A. B. H., \& Yassin, A. Y. (2014). Optimal sensor placement for mode shapes using improved simulated annealing. Smart Struct. Syst, 13(3), 389406. https://doi.org/10.12989/sss.2014.13.3.389.

Worden, K., \& Burrows, A. P. (2001). Optimal sensor placement for fault detection. Engineering Structures, 23(8), 885-901.

Yi, T. H., Li, H. N., \& Gu, M. (2011). Optimal sensor placement for structural health monitoring based on multiple optimization strategies. The Structural Design of Tall and Special Buildings, 20(7), 881-900. https://doi.org/10.1002/tal.712.

Zanotti Fragonara, L., Boscato, G., Ceravolo, R., Russo, S., Ientile, S., Pecorelli, M. L., \& Quattrone, A. (2017). Dynamic investigation on the Mirandola bell tower in post-earthquake scenarios. Bulletin of Earthquake Engineering, 15(1), 313337. https://doi.org/10.1007/s10518-016-9970-z.

Zawidzki, M., \& Jankowski, Ł. (2019). Multiobjective optimization of modular structures: Weight versus geometric versatility in a Truss-Z system. Computer-Aided Civil and Infrastructure Engineering, 34(11), 1026-1040.

Zhang, J., \& Sanderson, A. C. (2009). JADE: adaptive differential evolution with optional external archive. IEEE Transactions on evolutionary computation, 13(5), 945-958. https://doi.org/10.1109/TEVC.2009.2014613. 
$2021-02-17$

pÿA multi objective genetic algorithm strategy for robust optimal sensor placement

Civera, Marco

Wiley

pÿCivera M, Pecorelli ML, Ceravolo R, et al., (2021) A multi objective genetic algorithm strategy for robust optimal sensor placement. Computer-Aided Civil and Infrastructure Engineering,

Volume 36, Issue 9, September 2021, pp. 1185-1202

https://doi.org/10.1111/mice.12646

Downloaded from Cranfield Library Services E-Repository 